\title{
The clue of a possible etiology about spontaneous regression of hepatocellular carcinoma: a perspective on pathology
}

This article was published in the following Dove Press journal:

OncoTargets and Therapy

5 February 2015

Number of times this article has been viewed

\section{Zhuo Wangl,* \\ Zun-Fu Ke ${ }^{1, *}$ \\ Xiao-Fang Lu' \\ Can-Jiao Luo' \\ Yong-Dong Liu' \\ Zhong-Wei Lin² \\ Lian-Tang Wang'}

'Department of Pathology, The First Affiliated Hospital of Sun Yat-Sen University, Guangzhou, People's Republic of China;

${ }^{2}$ Department of Cardiology, The First Affiliated Hospital of Guangdong

Pharmaceutical University, Guangzhou,

People's Republic of China

*These authors contributed equally to this work
Correspondence: Lian-Tang Wang Department of Pathology, The First Affiliated Hospital of Sun Yat-Sen University, 58 Zhongshan Road II, Guangzhou, 5I 0080, People's Republic of China

Tel +86 $208733 \quad 1780$

Fax +86 2087331780

Email wanglt@mail.sysu.edu.cn
Abstract: Spontaneous regression of hepatocellular carcinoma (HCC) is a rare event. However, only a few of the causes of cases of HCC spontaneous regression are clear. More cases are ambiguous. We report on a patient who had a spontaneous regression of HCC as detected by histological and immunohistochemical exam, and compared this case to 20 cases of non-specific HCC. In our case, we found that the odd phenomenon is that $\mathrm{CD} 163^{+}$macrophages are overactivated in surviving HCC, which is spontaneously regressing. Concomitantly, we cannot find a similar phenomenon in peritumoral liver tissue or non-specific HCC. According to our microscopical morphology and immunohistochemical study, we considered that a clue of a possible etiology about $\mathrm{HCC}$ spontaneous regression is that $\mathrm{CD} 163^{+}$macrophages are overactivated.

Keywords: hepatocellular carcinoma, spontaneous regression, macrophages, CD163, CD68

\section{Introduction}

Spontaneous regression of cancer was first defined in 1959 by Cole and Everson as partial or complete disappearance of a malignant tumor in the absence of all treatment, or in the presence of therapy, which is considered to be inadequate to exert a significant influence on neoplastic disease. ${ }^{1}$ Malignant tumors that can convert to spontaneous regression included neuroblastoma, renal cell carcinoma, choriocarcinoma, liver cancer, etc. ${ }^{2-4}$ Unfortunately, spontaneous regression of hepatocellular carcinoma (HCC) is a rare event, ${ }^{5}$ and the underlying cause of this remission remains obscure.

Several mechanisms have been suggested to explain the etiology of spontaneous regression of HCC, including tumor ischemia, systemic inflammatory activation, temperance, drugs, and other mechanisms. ${ }^{6}$ Ischemia and inflammation are the main mechanisms. ${ }^{7}$ However, only $28.0 \%$ spontaneous regression of $\mathrm{HCC}$ can be attributed to tumor ischemia. ${ }^{7}$ Many regression cases (33.3\%) are due to a systemic inflammatory response, such as cholangitis and elevated cytokine levels. ${ }^{7-9}$ However, the histological diagnosis has not been done in most cases; in particular, the pathological operation resection has not been performed. This lack of diagnosis causes the systemic inflammatory response hypothesis to be insufficient.

In the present study, we report on a patient who had a spontaneous regression of HCC without any evidence of ischemia. We found the generating process of spontaneous regression of $\mathrm{HCC}$ in pathological examination after surgical resection.

\section{Materials and methods}

A patient was identified who had a spontaneous regression of $\mathrm{HCC}$ at The First Affiliated Hospital of Sun Yat-Sen University, Guangzhou, People's Republic of China. The 
patient's clinical features, plain computed tomography (CT), and pathological features were recorded. The patient with the spontaneous regression of HCC was then treated with surgical resection. The resected specimens were fixed in $10 \%$ buffered formalin and embedded in paraffin. An immunohistochemical study was performed using the following primary antibodies: CD68 (FLEX Ready-to-Use [RTU]; Dako Denmark A/S, Glostrup, Denmark); CD163 (RTU; Leica Microsystems, Wetzlar, Germany); CD20 (diluted 1:1,000; Dako Denmark A/S); CD79a (RTU; Dako Denmark A/S); CD3 (RTU; Dako Denmark A/S); CD5 (RTU; Dako Denmark A/S); CD4 (diluted 1:100; OriGene, Rockville, MD, USA); CD8 (diluted 1:500; Nobel Biocare, Zurich, Germany); CD56 (RTU; Dako Denmark A/S); Granzyme B (RTU; Dako Denmark A/S); and TIA-1 (RTU; Zeta Corp, Sierra Madre, CA, USA). Immunoreactivity was detected using the Dako-labeled streptavidin-biotin detection kit, according to the manufacturer's recommended procedures. Twenty cases of non-specific HCC were evaluated by immunological staining using CD68 and CD163 for comparison.

\section{Results}

\section{Clinical features of the patient with spontaneous regression of HCC}

A 50-year-old man was admitted to hospital with a 3-month history of slight abdominal discomfort. There was no evidence of recent fever, acute hepatitis, or drugs. There was no previous history of heavy alcohol intake, operations, or blood transfusions.

The patient was positive for serological markers of $\mathrm{HBV}$ (HBsAg+, HBsAb-, $\mathrm{HBeAg}-, \mathrm{HBeAb}+$, and $\mathrm{HBcAb}+$ ), but antibodies to HAV, HCV, HDV, HEV, and HGV were negative. The fetoprotein concentration level was $22,592.0 \mathrm{ng} / \mathrm{mL}$ (normal range, 0-9 IU/L). Investigations showed a normal white cell count. Aspartate aminotransferase activity was $53 \mathrm{IU} / \mathrm{L}$ (normal range, 8 40 IU/L); alanine aminotransferase activity was $70 \mathrm{U} / \mathrm{L}$ (normal range, 5 52 IU/L).

A liver tumor was first detected by ultrasonography. Ultrasonography showed isolated, large, highly reflective lesions suggestive of a tumor in the right lobe of the liver. Plain CT showed a nonuniform low-density area measuring $10.0 \mathrm{~cm}$ in diameter in Couinaud's segment 7 and 8 (S7 S8). Contrast-enhanced CT revealed the tumor to be partly encapsulated, enhanced for the most part, while it also had large low-density areas, suggesting necrosis (Figure 1). Thus, massive-type liver tumors in the right lobe were diagnosed as HCC. At the same time, the possibility of a prior ischemic event cannot be confirmed by angiographic data and CT.

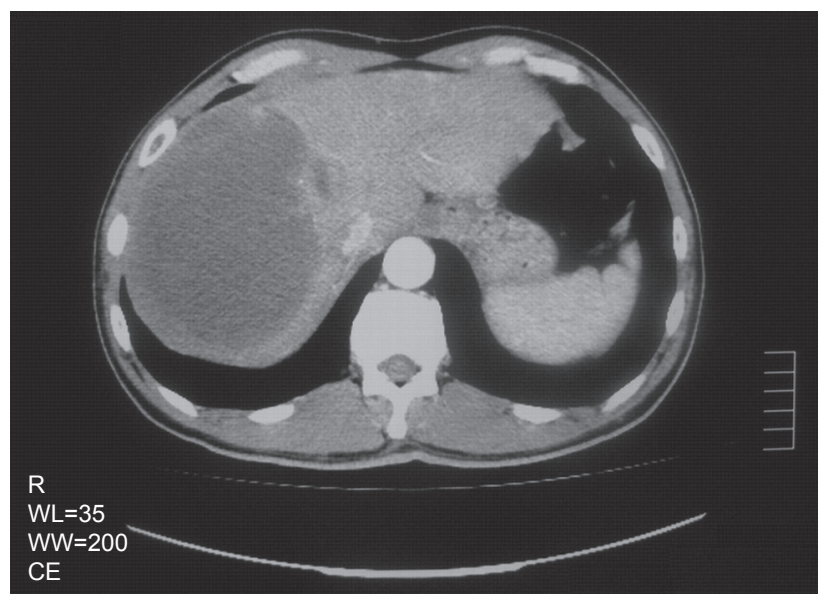

Figure I CT scan of the case of HCC spontaneous regression.

Notes: CT scan showed massive-type liver tumors located in the right lobe. They had a nonuniform low-density in Couinaud's segment 7 and 8.

Abbreviations: CT, computed tomography; HCC, hepatocellular carcinoma.

In May 2012, the patient underwent surgical resection by a right hepatectomy in the First Affiliated Hospital of Sun Yat-Sen University. About two years after surgery, he is still doing well and has no signs of recurrence.

\section{Histopathological and immunophenotypic analysis of the patient with spontaneous regression of $\mathrm{HCC}$}

Macroscopically, the tumor on the cut surface of the specimen, measuring 10×9 cm, was yellowish and brown with a partially fibrous capsule and septum-like structures (Figure 2). Microscopically, the tumor was an almost completely necrotic nodule with inflammatory cell infiltration, and was encapsulated by a fibrotic capsule. Hematoxylin and eosin

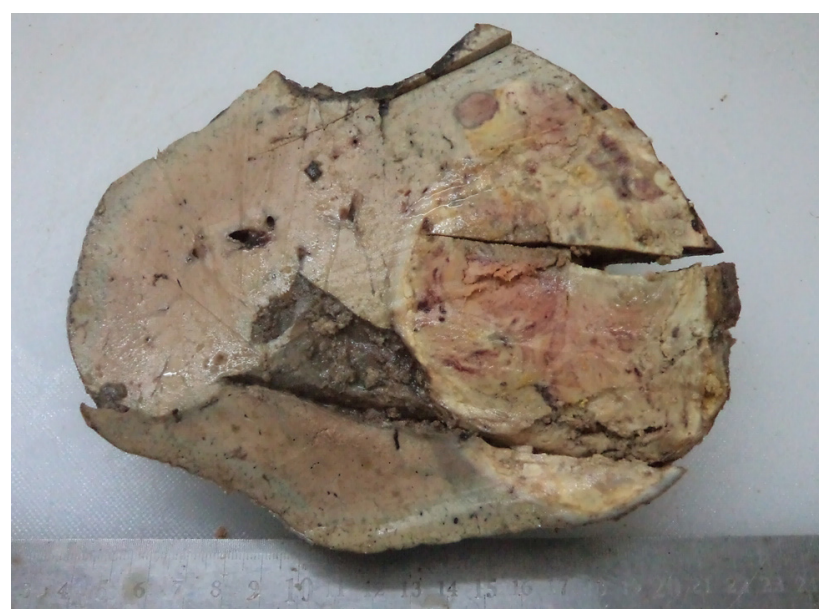

Figure 2 Macroscopical features of the case of HCC spontaneous regression. Notes: The tumor on the cut surface, measuring $10 \times 9 \mathrm{~cm}$, was yellowish and brown with a partially fibrous capsule and septum-like structures. Moreover, a reddishbrown nodule less than $1.2 \mathrm{~cm}$ was identified as surviving malignant tumor. Abbreviation: HCC, hepatocellular carcinoma. 
staining demonstrated that $99 \%$ of the tumor consisted of extensive coagulative necrosis without viable malignant cells; ghosts of the tumor cells were arranged in a trabecular pattern (Figure 3A).

Approximately $1 \%$ survival of the malignant zone was noted (Figure 3B, C). The surviving part of the tumor was composed of large polygonal cells arranged in sheets, and trabeculae. Moreover, many inflammatory cells infiltrated the periphery of the tumor (Figure 3D). A minority of inflammatory cells infiltrated the central zone of the partially surviving tumor. Most importantly, we found macrophages located between surviving portions of the tumor cells (Figure 3C). They had abundant cytoplasm and large nuclei. We showed these cells to be activated. Concomitantly, the background liver contained active chronic hepatitis and mild liver fibrosis. Thus, the diagnosis of this patient was mostly spontaneous regression of HCC.

The necrotic and surviving portions of the lesions revealed moderate lymphocytic and mononuclear cells reaction. An immunohistochemical study was performed, with the aim to analyze the inflammatory cells in the lesions. T cells $\left(\mathrm{CD}^{+}\right.$and $\left.\mathrm{CD}^{+}\right)$were more abundant than $\mathrm{B}$ cells $\left(\mathrm{CD} 20^{+}\right.$ and $C D 79 a^{+}$). There was little difference in the number of cells between $\mathrm{CD} 4^{+}$cells and $\mathrm{CD} 8^{+}$cells. However, immunophenotype analysis of CD56, Granzyme B, and TIA-1 were characterized by a lack of natural killer cells.
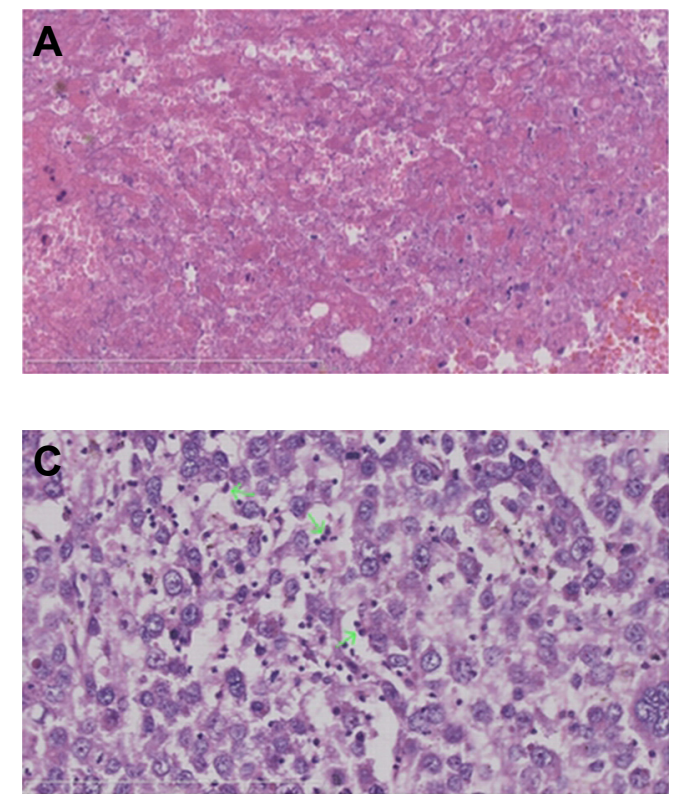

Immunological staining using CD68 demonstrated individual positive cells in the central zone of surviving portion of the tumor, although an amount of cells was also found in the fibrous capsule of the periphery of tumor (Figure 4A, B). However, an odd phenomenon is that the densities of intratumoral $\mathrm{CD} 163^{+}$cells were higher in partially surviving tumor than in the fibrous capsule and peritumoral liver tissue. Moreover, $\mathrm{CD} 163^{+}$cells had a larger size with abundant cytoplasm in partially surviving tumors (Figure 4C-E).

To confirm this odd phenomenon, 20 additional patients diagnosed with $\mathrm{HCC}, 20$ tissue samples from 20 other patients with HCC (including HCC with massive necrosis or HCC without necrosis) by immunological staining, using CD68 and CD163. Small amounts of $\mathrm{CD} 68^{+}$and $\mathrm{CD} 163^{+}$cells could be detected in non-specific HCC (Figure 5A, B). The density and size of $\mathrm{CD} 163^{+}$cells was lower in non-specific HCC than in the spontaneous regression of HCC (Figure 5C). The morphology of $\mathrm{CD} 163^{+}$cells was similar to those in peritumoral liver tissue in non-specific HCC (Figure 5D). Therefore, we suspected that highly activated macrophages in tumor were a possible cause of spontaneous regression of HCC. The highly activated macrophages are $\mathrm{CD} 163^{+}$.

\section{Discussion}

Spontaneous regression of HCC is well documented. But the cause is a medical enigma in most cases. Spontaneous
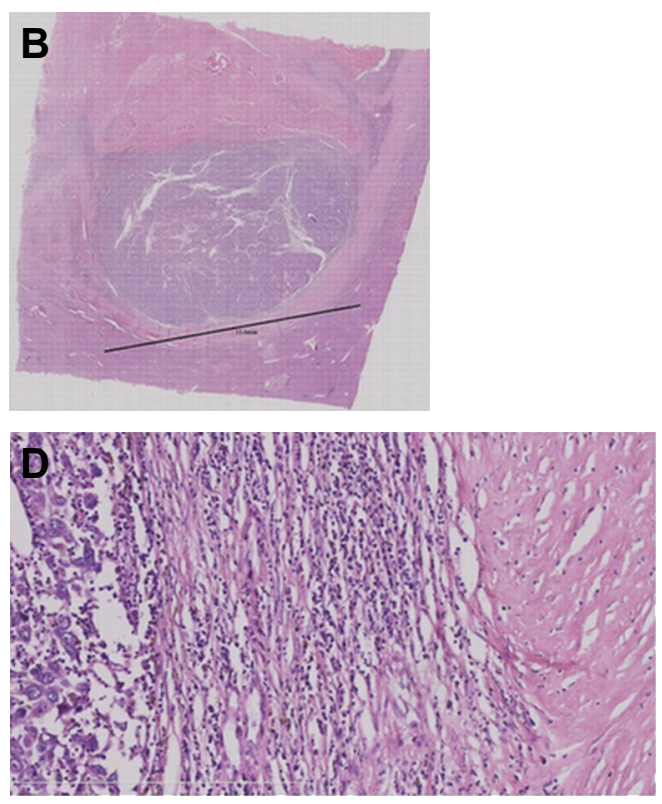

Figure 3 Microscopical features of the case of $\mathrm{HCC}$ spontaneous regression.

Notes: (A) Histologic examination shows coagulation necrosis of most of the zone in the tumor, and ghosts of the tumor cells are arranged in a trabecular pattern. (B) The surviving malignant zone was about $11.6 \mathrm{~mm}$ at low magnification. (C) At high magnification, the surviving part of the tumor was composed of highly pleomorphic cells arranged in sheets and trabeculae. Tumor cells are large and polygonal with central, vesicular nuclei and prominent nucleoli. Concurrently, some inflammatory cells infiltrate between tumor cells. The macrophages are the predominant inflammatory cells, with abundant cytoplasm and large nuclei (marked with the green arrows). (D) Many inflammatory cells infiltrated the periphery of tumor. Lymphocytes, neutrophils, and macrophages were detected.

Abbreviation: HCC, hepatocellular carcinoma. 

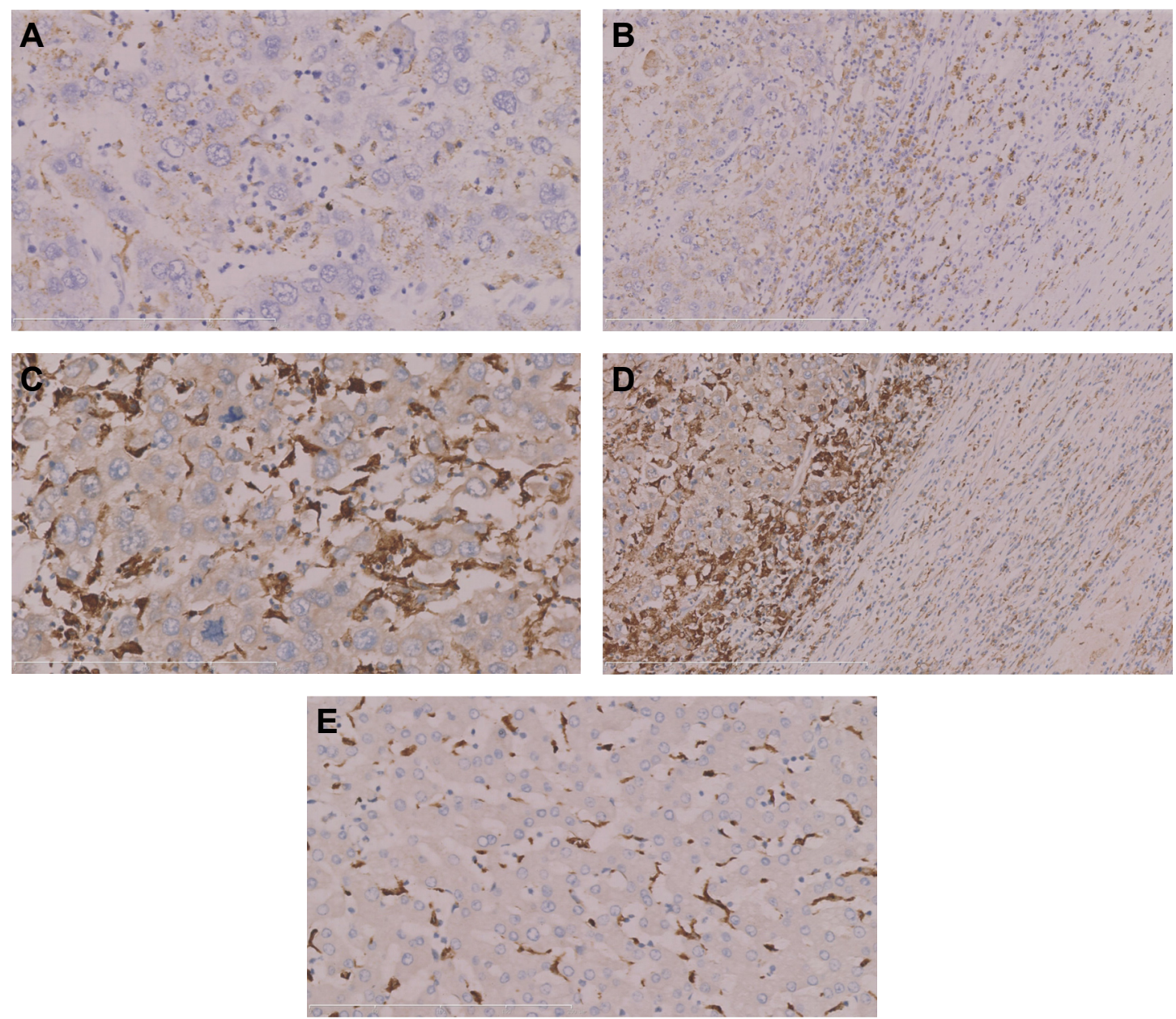

Figure 4 Immunological features of the case of HCC spontaneous regression.

Notes: (A) CD68 demonstrated individual positive cells in the central zone of the surviving portion of the tumor. (B) CD68 demonstrated an amount of positive cells in the fibrous capsule of the periphery of the tumor. The left part of the image is the tumor. The right part of the image is the periphery of the tumor. (C) CDI63 demonstrated more positive cells with large size and abundant cytoplasm in the central zone of partially surviving tumors. (D) CDI63 demonstrated many positive cells in the fibrous capsule of the periphery of the tumor. However, the positive cells in the fibrous capsule were smaller than in partially surviving tumors. The left part of the image is of partially surviving tumors. The right part of the image is the periphery of tumor. (E) CDI63 demonstrated a small amount of positive cells in peritumoral normal liver tissue.

Abbreviation: HCC, hepatocellular carcinoma.

regression of HCC may be due to tumor ischemia, such as hepatic artery thrombosis. ${ }^{10}$ The effect is similar to transcatheter arterial chemoembolization in the treatment of HCC. The inflammatory activation plays important roles in some cases of spontaneous regression of HCC. Determining the etiology of inflammatory reactions may help identify future therapeutic pathways for HCC immune-directed treatment.

In our case, we found that the odd phenomenon is that $\mathrm{CD} 63^{+}$macrophages are overactivated in surviving $\mathrm{HCC}$ that is spontaneously regressing. This is unusual. We cannot find a similar phenomenon in non-specific HCC. What explains this odd phenomenon? We considered two possible explanations. One explanation is that this overactivation is a reaction to tumoral necrosis, with scavenger receptor $\left(\mathrm{CD} 163^{+}\right)$upregulation commensurate with the increased cell volume and phagocytic activity. But this could not explain the fact that the overactivated macrophages $\left(\mathrm{CD} 163^{+}\right)$were most abundant in surviving tumors and lacking in the surrounding area of necrosis in our case. Moreover, we could not find the overactivated macrophages $\left(\mathrm{CD} 163^{+}\right)$in our 20 cases of non-specific HCC, especially HCC with massive necrosis. Another explanation is that overactivated macrophages $\left(\mathrm{CD} 163^{+}\right)$are the fundamental driving force behind spontaneous regression of HCC, although no other researchers have reported this discovery. We nonetheless prefer the latter explanation.

Most researchers accept that $\mathrm{CD}^{+} 8^{+}$is the most widely used marker for macrophages (pan-macrophages). Concomitantly, some reports have indicated that macrophages assume two different phenotypes based on environmental stimuli, the M1 (classically activated phenotype) 

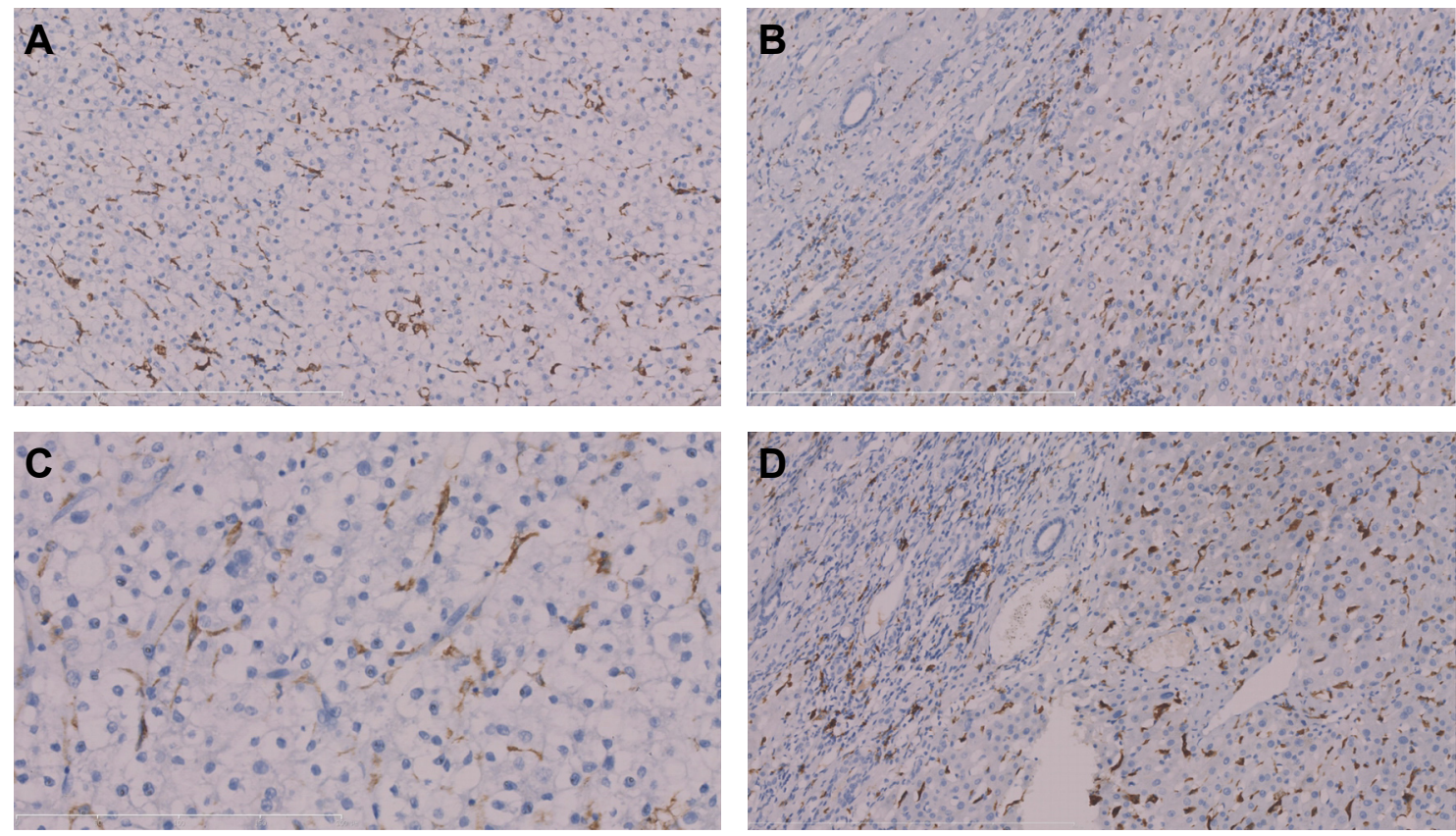

Figure 5 Immunological features of non-specific $\mathrm{HCC}$ as a control.

Notes: (A) CD68 demonstrated many positive cells in non-specific HCC, and (B) in the periphery of tumor. (C) CDI63 demonstrated many positive cells in non-specific $\mathrm{HCC}$, and (D) in the periphery of tumor, and the morphology of positive cells was similar. The density and size of both CD68 and CDI63+ cells were similar in nonspecific HCC.

Abbreviation: HCC, hepatocellular carcinoma.

and M2 (type II alternatively activated phenotype). CD68 could not distinguish between M1 and M2 subtypes. M2 macrophages have a high expression of several receptors such as CD163, which are polarized by anti-inflammatory molecules, such as interleukin-4 and interleukin-10, in order to show an immunosuppressive phenotype. ${ }^{11}$ CD163 is a glycoprotein belonging to the scavenger receptor cysteine-rich superfamily. CD163-expressing cells have a role in regulation of the immune response because they can be controlled by various inflammatory mediators. ${ }^{12}$ Recently, some researchers have paid attention to the role of macrophages in HCC, and have found that high abundance of macrophages was associated with poor prognosis of HCC. ${ }^{13}$ Other researchers postulated that serum $\mathrm{sCD} 163$ was a new prognostic parameter in HCC patients. ${ }^{14}$ Concomitantly, some research results showed that the local infiltration and plasma sCD163 of $\mathrm{CD}_{163^{+}}$cells were of limited significance in HCC, and that they were more likely markers related to active hepatitis rather than an indication of tumoral progression. ${ }^{15}$ We considered that there is a relationship between macrophages and poor prognosis in HCC, because a lot of macrophages infiltrated into peritumoral liver tissue, but not into tumors. This appearance was induced by the necrosis. Moreover, the activation of macrophages could release some cytokines, which leads to cell death. The balance between the necrosis and the infiltration of macrophages depended on different function of macrophages in tumors.

Furthermore, we observed in our case that the size of $\mathrm{CD} 163^{+}$cells in surviving tumors is obviously larger than the size of the same cells when they were located in peritumoral liver tissue. This prompted us to reason that the $\mathrm{CD} 163^{+}$cells in the surviving tumors of our case were activated when $\mathrm{CD}_{163}{ }^{+}$cells in peritumoral liver tissue were dormant. We considered that CD163 could not distinguish between activated and inactivated macrophages. Therefore, the assessments of the quantity of $\mathrm{CD} 163^{+}$cells in tumor tissue were defective. We look forward to the advent of new molecular markers to detect activated macrophages.

\section{Conclusion}

A rare case of spontaneous regression of $\mathrm{HCC}$, due mostly to necrosis, is presented in the present case report. In our case, we considered the overactivated macrophages $\left(\mathrm{CD} 163^{+}\right)$in tumors to be dominant, although there are many macrophages whose morphology and immunophenotype are different to the overactivated macrophages in peritumoral liver tissue and non-specific HCC. Therefore, we considered that possible etiology of $\mathrm{HCC}$ spontaneous regression is that $\mathrm{CD} 163^{+}$ macrophages are overactivated. 


\section{Disclosure}

The authors report no conflicts of interest in this work.

\section{References}

1. Cole WH, Everson TC. Spontaneous regression of cancer: preliminary report. Ann Surg. 1956;144:366-383.

2. Chan BP, Booth CM, Manduch M, Touma NJ. Spontaneous regression of metastatic pulmonary renal cell carcinoma in the setting of sarcomatoid differentiation of the primary tumour. Can Urol Assoc J. 2013;7: E587-E589.

3. Diede SJ. Spontaneous regression of metastatic cancer: learning from neuroblastoma. Nat Rev Cancer. 2014;14:71-72.

4. Bakyalakshmi K, Bharathi R, Ponniah I. A regressing and metastasizing tumor - the choriocarcinoma. J Oral Maxillofac Surg. 2013;71: 214-219.

5. Chiesara F, Spagnolo A, Koch M, Moretti A. A case of hepatocellular carcinoma: Spontaneous regression? Dig Liver Dis. 2014;46:659-660.

6. Okano A1, Ohana M, Kusumi F, Nabeshima M. Spontaneous regression of hepatocellular carcinoma due to disruption of the feeding artery. Case Rep Oncol. 2013;6:180-185.

7. Huz JI, Melis M, Sarpel U. Spontaneous regression of hepatocellular carcinoma is most often associated with tumour hypoxia or a systemic inflammatory response. HPB (Oxford). 2012;14:500-505.

8. Ohta H, Sakamoto Y, Ojima H, et al. Spontaneous regression of hepatocellular carcinoma with complete necrosis: case report. Abdom Imaging. 2005;30:734-737.
9. Heianna J, Miyauchi T, Suzuki T, Ishida H, Hashimoto M, Watarai J. Spontaneous regression of multiple lung metastases following regression of hepatocellular carcinoma after transcatheter arterial embolization. A case report. Hepatogastroenterology. 2007;54:1560-1562.

10. Hsu CY, Sun PL, Chang HC, Perng DS, Chen YS. Spontaneous regression of advanced hepatocellular carcinoma: a case report. Cases J. 2009; 2:6251.

11. Sulahian TH, Högger P, Wahner AE, et al. Human monocytes express $\mathrm{CD} 163$, which is upregulated by IL-10 and identical to p155. Cytokine. 2000;12:1312-1321.

12. Buechler C, Ritter M, Orsó E, Langmann T, Klucken J, Schmitz G. Regulation of scavenger receptor CD163 expression in human monocytes and macrophages by pro- and antiinflammatory stimuli. J Leukoc Biol. 2000;67:97-103.

13. Fujita N, Nishie A, Aishima S, et al. Role of tumor-associated macrophages in the angiogenesis of well-differentiated hepatocellular carcinoma: pathological-radiological correlation. Oncol Rep. 2014;31: 2499-2505.

14. Waidmann $\mathrm{O}$, Köberle $\mathrm{V}$, Bettinger $\mathrm{D}$, et al. Diagnostic and prognostic significance of cell death and macrophage activation markers in patients with hepatocellular carcinoma. J Hepatol. 2013;59:769-779.

15. Kong LQ, Zhu XD, Xu HX, Zhang JB, Lu L, Wang WQ, et al. The clinical significance of the $\mathrm{CD} 163^{+}$and $\mathrm{CD} 68^{+}$macrophages in patients with hepatocellular carcinoma. PLoS One. 2013;8:e59771.
OncoTargets and Therapy

\section{Publish your work in this journal}

OncoTargets and Therapy is an international, peer-reviewed, open access journal focusing on the pathological basis of all cancers, potential targets for therapy and treatment protocols employed to improve the management of cancer patients. The journal also focuses on the impact of management programs and new therapeutic agents and protocols on

\section{Dovepress}

patient perspectives such as quality of life, adherence and satisfaction. The manuscript management system is completely online and includes a very quick and fair peer-review system, which is all easy to use. Visit http://www.dovepress.com/testimonials.php to read real quotes from published authors. 